\section{La Révolution française}

Cahiers de l'Institut d'histoire de la Révolution française

$16 \mid 2019$

1801-1840 - Haïti, entre Indépendance et

Restauration

\title{
Bref aperçu concernant l'histoire du mouvement abolitionniste français (1770-1848)
}

\section{Bernard Gainot}

\section{CpenEdition}

\section{Journals}

Édition électronique

URL : http://journals.openedition.org//rf/3111

DOI : $10.4000 /$ Irf.3111

ISSN : 2105-2557

Éditeur

IHMC - Institut d'histoire moderne et contemporaine (UMR 8066)

Référence électronique

Bernard Gainot, « Bref aperçu concernant l'histoire du mouvement abolitionniste français (1770-1848)

», La Révolution française [En ligne], 16 | 2019, mis en ligne le 20 juin 2019, consulté le 22 juin 2019.

URL : http://journals.openedition.org//rf/3111 ; DOI : 10.4000/Irf.3111

Ce document a été généré automatiquement le 22 juin 2019

(c) La Révolution française 


\title{
Bref aperçu concernant l'histoire du mouvement abolitionniste français (1770-1848)
}

\author{
Bernard Gainot
}

\section{Introduction}

1 Vers le milieu du $\mathrm{XVIII}^{\mathrm{e}}$ siècle, la traite négrière transatlantique connaît un essor sans précédent qui va durablement transformer les sociétés coloniales et provoquer une forte croissance des façades maritimes de l'Europe occidentale. Ces changements matériels interpellent au premier chef les penseurs qui tiennent dans un espace public naissant un rôle actif et indépendant. Ils sont prisonniers d'un paradoxe : d'un côté, ils vantent les bienfaits du « doux commerce » qui rapproche les peuples, accroît le bien-être et assure la paix universelle. Mais, d'un autre côté, se développe la conscience du prix à payer pour ce progrès : la réduction en esclavage des populations africaines, l'horreur des cales des navires négriers, l'enfer des plantations. La demande d'une abolition de l'esclavage en Europe occidentale naît dans le sillage du progressisme des Lumières.

2 Mais l'abolitionnisme occidental est un mouvement d'essence universaliste. L'étude de la genèse des doctrines et des mouvements qui conduisirent à l'interdiction légale de l'esclavage permet de distinguer trois foyers distincts, puis convergents. Toutefois, aucun d'entre eux, même le plus radical, ne put assurer à lui-seul la destruction du système esclavagiste.

3 Le foyer originel est celui d'une résistance multiforme, et séculaire, des esclaves euxmêmes à leur réduction à l'état de machines: fuite, insurrection, recherche de l'autonomie par la culture (fêtes et danses), par la solidarité (associations), ou par l'entretien d'un lopin de terre familiale ${ }^{1}$.

4 Le deuxième foyer est celui des rivalités entre les puissances européennes, qui débouchaient sur des conflits armés. Les autorités coloniales étaient contraintes d'avoir recours à l'armement, total ou partiel, des esclaves. Mais, une fois le conflit terminé, les 
promesses d'affranchissement délivrées à ceux qui avaient pris les armes étaient oubliées, ou bien soigneusement limitées, et de nouvelles arrivées d'esclaves venaient encore renforcer le système esclavagiste ${ }^{2}$.

5 Enfin, le troisième foyer est celui de l'abolitionnisme doctrinal, qui va naître et se développer dans les principaux pays qui fondaient une grande partie de leur prospérité sur le développement de l'économie de plantation.

6 C'est ce dernier courant que nous nous proposons d'étudier plus particulièrement dans cette contribution. Après en avoir rappelé les origines transnationales, nous mettrons l'accent sur la spécificité de l'abolitionnisme français, qui se configure au cours de la décennie révolutionnaire et acquiert de ce fait un caractère radical. Sa " nationalisation » est donc liée à sa « radicalisation ».

7 Le Consulat ayant clos la parenthèse abolitionniste pour la France, c'est le jeune État d'Haïti qui recueille l'héritage révolutionnaire, ce qui ne manque pas de susciter de nombreuses interrogations lors de la renaissance d'un courant abolitionniste dans la France de la Restauration. Cette période, fort peu étudiée, est réduite par l'un des meilleurs spécialistes aux activités du Comité pour l'abolition de la traite des esclaves, organe de la Société de la morale chrétienne $e^{3}$. Cette focalisation sur l'une des composantes du mouvement, aussi importante soit-elle, peut donner l'impression que l'abolitionnisme français est à la remorque d'un milieu libéral protestant très minoritaire, lui-même dérivé de l'abolitionnisme anglo-saxon, qui aurait en quelque sorte placé l'abolitionnisme français sous tutelle. Le sentiment que l'on en retient est celui d'une faiblesse, d'une convalescence difficile dans un environnement hostile. Les abolitionnistes n'ont ni soutien populaire, ni sources de financement. Haïti, dans ce contexte, devient le point noir de l'héritage révolutionnaire. Le gradualisme très prudent qui tient lieu désormais de doctrine officielle tourne le dos au nouvel État.

8 À partir de cette lecture, selon moi partiale, et très partielle, de l'abolitionnisme renaissant de la Restauration, on peut en arriver à des jugements globaux et hâtifs. Évoquer Haïti, pour les gradualistes, constitue une gêne, dans la mesure où la conquête de la liberté générale s'est faite dans la conjoncture révolutionnaire : une abolition violente, immédiate, sans indemnité ${ }^{4}$. Une interprétation, aujourd'hui fréquemment admise, voudrait même que la révolution haïtienne soit cause d'une proclamation tardive de la seconde abolition, en 1848. Le lien entre révolution et abolition est interrogé. L'onde révolutionnaire favorise dans un premier temps la révolution des esclaves ${ }^{5}$, avec une présentation parfois singulièrement réductrice de la réalité, puis, sur la moyenne durée, « l'abolitionnisme fut en France rapidement associé par ses opposants à l'idée de Terreur et à la perte de Saint-Domingue attribuée aux propos des abolitionnistes. D'où un divorce entre abolitionnisme et idée nationale, mais aussi les réticences des milieux abolitionnistes ultérieurs vis-à-vis de l'action populaire ${ }^{6} »$.

9 Je propose ainsi, dans une seconde partie, de mettre l'accent sur la spécificité d'un abolitionnisme français, toujours ressourcé dans la conservation de rapports privilégiés avec l'État indépendant d'Haïti ${ }^{7}$. Cet héritage conservé est ouvertement revendiqué dans les années 1840 , lorsque l'abolitionnisme renoue avec ses inspirations républicaines. 


\section{Internationalisme et rupture républicaine. La constitution d'un héritage (1770-1800).}

\section{Un internationalisme de principe.}

D'où vient donc ce courant abolitionniste, qu'il faut appréhender de prime abord comme un mouvement international, qui se développe dans l'espace atlantique concerné par la traite négrière et l'économie de plantation?

11 Il a tout d'abord une origine religieuse. Alors que les Églises établies, catholiques comme protestantes, s'accommodent très bien de l'esclavage, une opposition interne a toujours existé qui affirme tout à la fois l'unité du genre humain (monogénisme) et la liberté naturelle de tout être humain.

En Angleterre, ces opposants fuyaient les persécutions de l'Église établie en se réfugiant dans les colonies américaines. C'est au sein de l'un de ces courants dissidents, les Quakers, que, outre le principe interne qu'on ne pouvait posséder un esclave et être membre de l'Église quaker, va naître un programme pour obtenir une loi générale d'abolition de l'esclavage dans la colonie de Pennsylvanie. Antony Benezet est l'auteur d'un des premiers textes anti-esclavagistes, publié en 1771 à Philadelphie : Some historical account of Guinea, its situation, produce and the general disposition of its inhabitants. With an inquiry into the rise and progress of the slave-trade, its nature and lamentable effects (... ${ }^{8}$. En 1775 , il fonde une Société de secours aux nègres libres illégalement maintenus en servitude, qui est la première variante des futures sociétés des Amis des Noirs.

Après 1776 et le développement de la Guerre d'Amérique, le mouvement va s'étendre à tout le courant contestataire anglais, qui se nomme les dissenters. Ces protestants en rupture d'église réclament un retour à la pureté originelle de la doctrine chrétienne (les évangéliques) laquelle, selon eux, affirme l'incompatibilité entre l'esclavage et la foi.

En France, un courant de même nature existe au sein de l'Église catholique. Quoique moins répandu, c'est tout de même l'inspiration de l'un des abolitionnistes français les plus notoires, l'abbé Grégoire. Il existe de même des pôles individuels religieux de refus de l'esclavage en Espagne, sur le modèle du père Juan Pedro Claver à Carthagène, au XVII ${ }^{\mathrm{e}}$ siècle, qui baptisait les captifs à leur arrivée en Amérique, pour poursuivre ensuite en justice propriétaires et négriers, en invoquant l'impossibilité de réduire un chrétien à la servitude ${ }^{9}$.

15 Mais la principale source doctrinale en France est laïque. Déjà, au XVI ${ }^{\mathrm{e}}$ siècle, dans les Six livres de la République, ouvrage publié en 1576, Jean Bodin avait réfuté les arguments d'Aristote, qui pensait que l'esclavage était dans l'ordre des choses (l'esclavage par nature ). Selon Bodin, l'esclavage est contre nature. Mais il écrit alors dans un pays, la France, où l'esclavage est interdit depuis 1317. Une ordonnance royale stipule qu'aucun esclave ne peut exister sur le sol français. La France n'a pas de colonies ultra-marines à cette époque. Par la suite, l'argument servira essentiellement à la condamnation de la réduction en esclavage des Amérindiens et toute une controverse s'élève à ce sujet. Si les esclavagistes ne peuvent plus guère soutenir la légitimité de l'esclavage des indigènes par l'infériorité de leur nature, ils font découler celle-ci du droit de la guerre : il est plus humain de contraindre les vaincus au travail forcé que de les mettre à mort. 

sur un plan général, universel, en liant très clairement la déshumanisation de la condition servile à la mise en esclavage des Noirs dans les territoires où l'économie de plantation s'est généralisée. Dans De l'esprit des lois, en 1748, Montesquieu réfute les arguments des penseurs qui admettaient l'esclavage par droit de guerre et de conquête. Pour lui, «l'esclavage est le plus violent fait de la nature humaine.» Et, pour se faire bien comprendre, il ajoute : «La guerre de Spartacus était la plus légitime qui ait jamais été entreprise. » Une décennie plus tard, en 1762, Rousseau affirmera l'incompatibilité entre l'état de droit et l'esclavage. Dans L'Encyclopédie, Jaucourt, à l'article «Esclave», est extrêmement catégorique dans sa conclusion: «L'esclavage, fondé par la force, la violence, et, dans certains climats, par excès de la servitude, ne peut se perpétuer que par ces mêmes moyens. » Un grand pas était franchi du point de vue doctrinal ${ }^{10}$. Il préparait l'abolition par la loi.

La troisième source, c'est l'économie politique. Dans les années 1770 , une partie des économistes qui réfléchissent sur les rendements comparés du travail libre (salarié) et du travail servile, conclut à la nocivité de l'économie esclavagiste de plantation; inutile parce qu'elle détruit l'intérêt du travail productif, perverse parce qu'elle dégrade l'humanité et du maitre et de l'esclave. Dupont De Nemours, dans un article du journal les Éphémérides du citoyen en 1771, propose un nouveau programme de substitution à cette économie toxique ${ }^{11}$. Turgot, en 1774, pense que l'esclavage dans les Antilles est condamné à terme. Adam Smith, en 1776, dans La richesse des nations, affirme pour tous les temps et tous les lieux la supériorité du travail libre sur le travail servile.

Un ouvrage va faire la synthèse de toutes ces sources doctrinale, morale, économique et philosophique, c'est l'Histoire philosophique et politique des établissements européens dans les Deux Indes, un best-seller de l'édition du xviII ${ }^{\mathrm{e}}$ siècle, traduit rapidement dans tous les pays européens du monde occidental. L'abbé Raynal prête son nom à une entreprise qui regroupe des auteurs différents; sous ce nom collectif, Diderot lance un avertissement radical. Si des réformes ne sont pas rapidement entreprises pour mettre fin à la traite des africains et à l'exploitation du travail des esclaves, un Spartacus noir prendra la tête d'une formidable révolte des esclaves du Nouveau Monde ${ }^{12}$.

\section{La « cristallisation » : le militantisme abolitionniste}

Dans les États du nord de l'Amérique devenus indépendants en 1783, les comités abolitionnistes et les sociétés anti-esclavagistes se multiplient à Philadelphie, New-York, Boston... En Angleterre, les initiatives dispersées sont rassemblées en mai 1787 autour de la Society for effecting the abolition of the slave trade. Ses principaux fondateurs sont les quakers Granville Sharp et Thomas Clarkson, rejoints par le parlementaire William Wilberforce.

20 Le banquier d'origine genevoise Étienne Clavière, ainsi que le journaliste français JeanPierre Brissot font des voyages aux États-Unis et en Angleterre, prenant des contacts avec ces comités abolitionnistes. En février 1788 est fondée à Paris la Société des Amis des noirs sur le modèle britannique, et en étroite correspondance avec la société-mère de Londres. Elle regroupe un peu plus d'une centaine de membres au cours des deux premières années de la Révolution ${ }^{13}$. Brissot et Clavière sont rejoints, entre autres, par l'abbé Grégoire, le pasteur Frossard, le docteur Lanthenas, le philosophe économiste Condorcet, 
et de nombreuses personnalités politiques de premier plan comme Mirabeau, La Fayette et La Rochefoucauld-Liancourt.

21 L'objectif est d'obtenir, par une action concertée entre les puissances européennes impliquées dans le commerce triangulaire - France, Angleterre, Danemark, en premier lieu, puisque ce sont les pays où s'est développée une structure abolitionniste -, une interdiction légale et universelle de la traite négrière. Puis des réformes seront entreprises dans le régime de l'esclavage - fin des châtiments corporels, repos des fins de semaine, reconnaissance d'un statut personnel juridique pour mener des actions en justice. Les affranchissements individuels seront facilités, avec la perspective d'un affranchissement général à terme, toujours en coordination avec les autres puissances européennes. Cette abolition graduelle serait longue : deux ou trois générations.

Les moyens d'action sont de deux ordres: d'abord, en direction de l'opinion publique, pour populariser la cause par des pétitions, des brochures, des symboles. En Angleterre, Thomas Clarkson se lance dans une enquête approfondie, sur le terrain, dans les ports anglais qui vivaient du commerce des esclaves, pour recueillir un maximum d'informations et de témoignages sur la barbarie de la traite négrière. Pour concrétiser les résultats fut élaborée une maquette d'un navire négrier de Liverpool, le Brooks, qui révélait les conditions d'entassement et de proximité du transport de la cargaison humaine. La maquette fut présentée à l'Assemblée nationale par Mirabeau en décembre 1789.

Un sigle, élaboré par le manufacturier Josiah Wedgwood, va populariser la cause abolitionniste et assurer sa visibilité dans l'espace public : un Noir agenouillé et enchaîné implore les témoins compatissants "Am I not a man and a brother? », formule traduite (presque) littéralement dans la version française: «Ne suis-je pas un Homme et ton Frère?».

24 Le deuxième moyen d'action est de faire pression sur les autorités constituées: le Ministère de la Marine et des colonies, tout d'abord, où le ministre De Castries était très ouvert aux projets de réformes au cours des années postérieures à la Guerre d'Amérique ; l'Assemblée Nationale ensuite, où la Société des Amis des Noirs se heurtait au Comité colonial dominé par les partisans du statu quo esclavagiste.

En Angleterre, le mouvement est porté jusqu'à l'Assemblée et au Ministère par des personnages comme Wilberforce, soutenu par le premier ministre William Pitt. En avril 1791, Wilberforce propose un projet de loi pour abolir la traite négrière qui est repoussé par 163 voix contre 88 .

Outre la traite atlantique, la France est également concernée par la traite de l'océan Indien, entre l'Afrique orientale (Mozambique), Madagascar, et les Mascareignes (île Bourbon et île de France, surtout, où l'économie esclavagiste connaît un développement spectaculaire depuis 1760).

\section{La rupture républicaine. L'abolitionnisme radical.}

Le cours de l'Histoire et, singulièrement, celui de la Révolution française, vont faire diverger cet internationalisme initial. L'abolitionnisme français s'engage dans une voie plus radicale.

28 Les priorités vont changer. Tandis que l'action internationale concertée s'éloigne avec la montée des tensions, les résistances aux projets de réformes s'organisent aussi bien en 
métropole que dans les assemblées coloniales. Obtenir le changement par la loi devient plus difficile, alors que la stratégie des Amis des Noirs était essentiellement fondée sur la recherche $\mathrm{du}$ consensus: obtenir des autorités une extension des droits et une humanisation des conditions, faire admettre aux négociants que leur intérêt réside dans la progressive généralisation du métayage ou du salariat.

Mais les planteurs s'accrochent au statu quo, tandis que les couches populaires blanches recourent à la violence pour défendre le préjugé de couleur, et se protéger des revendications égalitaires portées par les libres de couleur (Noirs affranchis et mulâtres). Il paraît vite impossible de vaincre ces résistances, sans établir un rapport de forces. Les libres de couleur sont alors identifiés comme les véritables soutiens d'un projet révolutionnaire. Le particularisme colonial s'estompe, du moins aux Antilles, car il reste dominant aux Mascareignes.

30 Progressivement, la révolution des couleurs converge avec le cours de la révolution en métropole. Non sans contradictions, qui expliquent la cessation des activités de la Société des Amis des Noirs en 1791. Mirabeau est mort en avril 1791, les réformistes comme La Fayette prennent leurs distances avec l'évolution radicale et populaire de la Révolution, tandis que les plus engagés, comme les brissotins, sont absorbés par les responsabilités nationales et les luttes politiques internes.

31 Aux Antilles, les troubles civils se généralisent, tandis que le conflit international à partir de 1793 vient anéantir le programme de l'abolition graduelle et concertée. C'est une autre voie qui est empruntée, celle de l'abolition immédiate et révolutionnaire. Le décret adopté par la Convention nationale le 16 pluviôse an II (4 février 1794) pour établir la liberté générale ne peut se comprendre que comme une convergence des trois contextes, dissociés à l'origine: l'imposition d'un état d'exception pour sauver la République menacée par les deux puissances esclavagistes rivales (Angleterre, Espagne), l'armement général des populations de couleur, esclaves et libres confondus, et la mise en conformité des lois et des principes doctrinaux - abolition universelle de l'esclavage, loi de la majorité, donc fin des discriminations fondées sur la couleur, et fin du particularisme colonial avec l'égalité de tous les citoyens devant la loi (principe d'isonomie, ce qui veut dire la même loi pour toutes les provinces).

Le décret ne fut appliqué que dans les territoires conservés par la République française aux Antilles, Saint-Domingue, Guadeloupe et Guyane. Il fut rejeté par les colons des Mascareignes, qui vécurent de ce fait pendant quatre ans en état de rébellion.

Si la singularité révolutionnaire de la mesure, assimilée à de la précipitation, a pu être regrettée par la suite par une fraction significative du mouvement abolitionniste (Grégoire notamment), elle ne fut jamais remise en cause par ses partisans, ni par les autorités officielles du Directoire. L'abolition est un acquis de la République, inscrit dans la Constitution de 1795, garanti par l'assimilation des anciennes colonies aux départements métropolitains. La Société des Amis des Noirs allait renaître de 1797 à 1799 autour de Grégoire, Lanthenas, Laveaux, Jean-Baptiste Say, Carl-Bernard Wadstroem et des nombreux représentants de couleur envoyés par les colonies siéger aux Conseils du Directoire. Elle regroupera quatre-vingt-douze membres, dont quelques femmes ${ }^{14}$, autour d'un programme dont le premier volet est la défense et l'extension du décret sur la liberté générale par la "régénération" des anciennes colonies à esclaves. Ce terme de " régénération » signifie la transformation des anciens esclaves en " nouveaux citoyens ", par l'éducation, l'état-civil unique (acquisition d'un nom patronymique) et la rémunération du travail (une portion variable du revenu des plantations était affectée au 
salaire, une autre portion à l'impôt, la dernière portion à l'investissement). Le deuxième volet du programme est l'abolition internationale de la traite négrière. Dans cette perspective, la Société charge une commission recrutée en son sein de traduire les ouvrages étrangers dénonçant le commerce négrier, afin de préparer une campagne qui serait relayée au niveau des Congrès diplomatiques internationaux. Enfin, la dernière perspective est la recherche d'une nouvelle forme de rapports avec les peuples nonEuropéens, appelée "nouvelle colonisation». Des "établissements libres » doivent se multiplier en Afrique, puis, progressivement, dans toutes les régions d'où étaient originaires les esclaves, sur le modèle de la Sierra-Leone, encadré par les abolitionnistes Anglais dès $1787^{15}$.

Une fête annuelle marque la commémoration du décret du 16 pluviôse. Elle est officialisée dans les îles restées françaises de la Caraïbe, au même titre que les autres fêtes républicaines.

Ce renouveau et ces projets s'effondrent en même temps que le Directoire. Le nouveau régime issu du coup d'État de Brumaire, conduit par Bonaparte, mène une offensive brutale de très grande conséquence contre l'abolitionnisme (significativement qualifié de nigrophilisme), tenu pour responsable de la ruine des colonies. En réalité, cette offensive idéologique de grande ampleur s'inscrit dans un mouvement plus large de réaction contre l'héritage des Lumières. Elle accompagne la destruction de toute la législation ultramarine de la Révolution: l'abandon du décret sur la liberté générale et le retour à l'exception coloniale (décembre 1799), le rétablissement de l'esclavage à la Guadeloupe et en Guyane (1802-1803), l'échec de l'expédition militaire à Saint-Domingue et l'indépendance d'Haïti (1802-1804).

36 Le bilan de la période 1789-1815 est donc fortement contrasté, puisqu'elle a vu se succéder des politiques contradictoires: de l'abolitionnisme le plus radical avec ses anticipations égalitaires jusqu'à un régime d'assignation des catégories ethno-juridiques à leurs origines raciales, beaucoup plus violent et figé que le simple rétablissement de la société esclavagiste et ségréguée d'Ancien Régime ${ }^{16}$. Sur ce dernier point, il y a une remarquable continuité entre la politique coloniale officielle de l'Empire napoléonien et celle de la Restauration. Mais cette politique est également maintenue, voire renforcée, dans les colonies qui passent épisodiquement (Martinique, Guadeloupe, Guyane, Réunion) ou définitivement (Maurice) sous occupation britannique.

\section{L'impossible oubli de l'héritage républicain. La voie française, c'est l'immédiatisme (1815-1848).}

\section{Une lente renaissance de l'internationalisme}

Les abolitionnistes sont réduits au silence ou à l'exil pendant l'Empire. Seule, la voix du sénateur Grégoire s'élève en 1808 dans La littérature des nègres pour rappeler les combats passés et les multiples contributions des Africains à la civilisation universelle, à une époque où les doctrines officielles les tiennent au mieux pour totalement sauvages, au pire comme étrangers à l'espèce humaine ${ }^{17}$.

38 Sur les rives du lac Léman, dans sa résidence de Coppet, la fille de Necker, madame De Staël, réunit quelques penseurs libéraux attachés à la préservation de l'héritage des Lumières qui voyait une absolue contradiction entre l'esclavage et le droit. D'origine 
suisse, installé en Angleterre, proche d'Helen-Maria Williams, qui fut une personnalité éminente de la seconde Société des Amis des Noirs, le peintre romantique JohannHeinrich Füssli expose en 1807 un sombre tableau qui est un écho des prédictions de Diderot-Raynal et des révoltes anti-esclavagistes de la période révolutionnaire : Le Nègre vengé ou la complainte du Noir.

Dans ce contexte déprimé, ce sont les Anglais et les États-Uniens qui reprennent l'initiative. La loi de prohibition de la traite négrière (Slave Trade Act) est acceptée à la Chambre des Communes par une large majorité de 283 voix contre 16, puis sanctionnée par le roi le 25 mars 1807. C'est une revanche pour Wilberforce, conservateur sur le plan politique, hostile à l'abolition immédiate de l'esclavage, invoquant le précédent révolutionnaire français comme une catastrophe. Le gouvernement fédéral des États-Unis suit le $1^{\text {er }}$ janvier 1808 en interdisant l'importation d'esclaves sur son territoire. La mesure est universalisée au Congrès de Vienne de 1815, mais peu de mesures concrètes seront mises en œuvre pour la faire appliquer.

La fin de l'Empire relance d'ailleurs le thème de l'abolitionnisme. Sur la question coloniale, la doctrine officielle est non seulement le retour à la situation qui prévalait antérieurement à 1789 , mais aussi l'oubli volontaire des faits se rapportant à la période révolutionnaire, dont l'abolition de l'esclavage, sous prétexte de préservation de la paix civile. L'affaiblissement du régime entraîne une libération de la parole et de l'écrit, dans les deux sens. Les abolitionnistes se concentrent sur la prohibition internationale de la traite négrière. Ce sont les libéraux du "groupe de Coppet", autour de Germaine De Staël, Benjamin Constant et Jean-Charles Sismonde De Sismondi, qui sont à la pointe du mouvement ${ }^{18}$. En 1814, Sismondi fait paraître un Essai sur les désavantages politiques de la traite des nègres, tandis que Germaine De Staël lance un Appel aux souverains réunis à Paris pour en obtenir l'abolition de la traite des nègres. En février 1815, les ambassadeurs plénipotentiaires des puissances européennes réunis en congrès international à Vienne signent une déclaration commune selon laquelle «le commerce connu sous le nom de traite des nègres d'Afrique a été envisagé par les hommes éclairés de tous les temps comme répugnant aux principes d'humanité et de morale universelle ${ }^{19} \%$. Chaque gouvernement restait toutefois pleinement souverain quant au délai de mise en application des mesures effectives de répression de la traite. Talleyrand s'engage au nom du gouvernement des Bourbons, mais celui-ci est renversé par le retour de Napoléon en mars 1815. Tardivement rallié, Benjamin Constant influence la rédaction du décret du 29 mars 1815 qui interdit la traite, mais cette disposition n'est pas reprise dans l'Acte additionnel aux constitutions de l'Empire du mois suivant.

Le retour définitif des Bourbons après Waterloo (juin 1815) ne permet aucunement de concrétiser ces timides avancées. Bien au contraire, le gouvernement de la Restauration est soumis à la pression du lobby colonial pro-esclavagiste, qui compte plusieurs de ses représentants les plus éminents parmi les ministres, dont Malouet à la Marine et aux colonies, et Vaublanc à l'Intérieur. Une campagne d'opinion bien orchestrée réclame tout à la fois la fin de la parenthèse indépendantiste d'Haïti ${ }^{20}$, par la force (une nouvelle expédition militaire) ou par la division (gagner les élites mulâtres contre la classe dirigeante noire), et la relance à grande échelle de la traite négrière, seule garante du développement économique des façades maritimes ruinées par le blocus.

La Restauration voit un lent réveil de l'abolitionnisme graduel. L'esclavage est maintenu dans les six colonies que la France a récupérées: Martinique, Guadeloupe, Guyane, Réunion, Saint-Louis du Sénégal et Gorée. Il se renforce même, alimenté par une traite 
illégale, dont la déréglementation autorise tous les excès. En dépit d'un respect formel des dispositions du Congrès de Vienne (décret du 20 novembre 1815), les années de la Restauration sont celles d'une envolée de la traite clandestine : 77500 esclaves sont introduits en Guadeloupe et à la Martinique entre 1815 et $1831^{21}$.

C'est donc en priorité à cette traite clandestine que s'en prennent les mouvements abolitionnistes renaissants au début du XIX ${ }^{e}$ siècle. Le courant abolitionniste anglais fait de nouveau figure de modèle et d'inspirateur, comme au cours de la période qui précéda la Révolution Française. Il est même possible de parler alors d'un véritable magistère moral et pratique des abolitionnistes anglais. Ils envoient des délégués, financent des publications, donnent des conseils pour la multiplication des comités anti-esclavagistes. Cet activisme d'inspiration puritaine n'est pas sans risques pour les abolitionnistes français et peut expliquer, pour une bonne part, leur isolement initial : image d'un « parti de l'étranger ", relais du prosélytisme protestant dans une France travaillée par la renaissance catholique. Mais le plus déterminant est le moule uniforme dans lequel vont être coulés les mouvements abolitionnistes frères. C'est le gradualisme, le remplacement progressif de l'esclavage par le métayage. Il s'agit d'un apprentissage de la liberté, pour une durée comprise entre six et soixante ans, les anciens esclaves ayant acquis la personnalité juridique étant placés sous la tutelle de leurs anciens maîtres et des autorités. C'est ce système qui est instauré dans les colonies anglaises en 1833.

La Société de la morale chrétienne, fondée en décembre 1821, compte 388 membres identifiés. Elle a créé en son sein, le 8 avril 1822, un comité pour l'abolition de la traite des Noirs, sur les directives d'un quaker anglais, Joseph Price ${ }^{22}$. Zachary Macaulay, éditeur du journal Antislavery Reporter, est présent à la fondation. Il est également l'intermédiaire pour les transferts de fonds servant à l'édition et à la diffusion de brochures et de mémoires. Le comité, qui comporte dix-sept membres, est présidé par Auguste De Staël, le fils de Germaine De Staël. Ses membres sont majoritairement des protestants, mais aussi des abolitionnistes de la première heure comme La Fayette, Grégoire, Frossart ou Lanjuinais. Outre Auguste De Staël, le groupe dirigeant comprend le pasteur AthanaseCharles Coquerel, l'écrivain Charles De Rémusat (qui fait jouer en 1825 la pièce dramatique L'Habitation de Saint-Domingue ou l'insurrection), les philanthropes Joseph De Gérando, Alexandre De Laborde, Charles Lasteyrie du Saillant. Il s'élargit ensuite pour accueillir Benjamin Constant, le banquier Benjamin Delessert, Hippolyte Carnot, ou encore François Guizot. Tous ces noms rattachent la cause abolitionniste à celle de l'opposition libérale envers le cours de plus en plus rétrograde pris par la monarchie restaurée. Les initiatives de la Société étaient d'ailleurs relayées dans la presse libérale (par exemple la Revue Encyclopédique de Marc-Antoine Jullien, ou La Minerve française de Benjamin Constant), qui regroupait l'opposition de gauche à la monarchie des Bourbons. La touche protestante initiale est cependant toujours bien présente. En mai 1825 naît un Comité des Dames de Paris, ou Société auxiliaire des femmes en faveur de l'œuvre des missions évangéliques chez les peuples non-chrétiens. Cette initiative est encouragée par les abolitionnistes britanniques et par les mêmes milieux protestants présents à la Société de la morale chrétienne ${ }^{23}$. Les actions principales sont dirigées contre la traite des africains, clandestine, mais couverte par les gouvernements de la Restauration. En 1824, par exemple, Auguste De Staël dirige une grande enquête à Nantes pour dénoncer les armements négriers. Cette mission sur le terrain rappelle également l'enquête de Thomas Clarkson dans les ports anglais à la fin du siècle précédent. Concrétisant une demande exprimée dans le testament de l'abbé Grégoire, le comité attribuait en outre des prix 
annuels, baptisés du nom de l'abbé, destinés à récompenser l'ouvrage qui défendait le mieux la cause abolitionniste.

\section{Affirmation d'une voie nationale : libéralisme politique, centralité de la question des libres de couleur, légitimité de Haïti.}

Progressivement, par des actions pratiques diverses qui contribuent à sortir la cause abolitionniste des seuls manifestes propagandistes, le comité s'oriente vers une structure plus nettement anti-esclavagiste : dénonciation de cas de maltraitance, rachat progressif des femmes esclaves qui, par l'affranchissement graduel, permettrait une émancipation progressive de la main d'œuvre servile. Ces actions sont d'ailleurs mises en œuvre aussi bien par le Comité pour l'abolition de l'esclavage, que par le Comité des Dames de Paris. Par ailleurs, des filiales voient le jour dans quelques grandes villes portuaires (Bordeaux, Marseille) ou industrielles marquées par le patronat protestant (Mulhouse, Nîmes).

Certes, il s'agit toujours de préparer un affranchissement graduel, selon le modèle proposé par le pasteur Charles Coquerel: les esclaves rachètent progressivement leur liberté en travaillant trois jours par semaine pour épargner. Et, aussi centrale soit-elle, la Société de la morale chrétienne n'épuise pas la totalité des initiatives opposées à la structure coloniale esclavagiste bien implantée au cœur du pouvoir. Les grands traités dénonciateurs renouent avec la période de la fin de l'Ancien Régime, comme le plus célèbre d'entre eux, le Précis historique de la traite des Noirs et de l'esclavage colonial contenant l'origine de la traite, ses progrès, son état actuel et un exposé des horreurs produites par le despotisme des colons, paru en 1828, dont l'auteur est un fonctionnaire de l'administration coloniale, et savant orientaliste spécialiste des langues indo-européennes, Joseph-Elzéar Morenas. Révolté par son passage dans les comptoirs sénégalais, qui sont, à l'époque de la Restauration, des observatoires privilégiés pour la traite clandestine et les expéditions en direction de l'Afrique intérieure, Morenas avait précédemment lancé des pétitions réclamant l'interdiction effective du commerce des esclaves ${ }^{24}$.

L'ancienne Saint-Domingue, devenue Haïti indépendante, république réunifiée sous le régime autoritaire du président Boyer, gouvernée par une élite de couleur, est revenue au cœur du débat public avec la question de la reconnaissance diplomatique par son ancienne métropole. Ce faisant, dans la floraison de publications ravivant la mémoire de l'expédition désastreuse lancée par Bonaparte pour reconquérir l'île, les plus marquantes (Pamphile de Lacroix ${ }^{25}$, Antoine Métral ${ }^{26}$ ) questionnent la légitimité du combat des insurgés contre l'esclavage et le système colonial, reprenant les mises en garde du colonel Malenfant en 1814 contre une nouvelle aventure aux Caraïbes ${ }^{27}$. Mais nul ne va aussi loin que Civique De Gastine ${ }^{28}$, pseudonyme de l'ancien activiste babouviste Eustache Toulotte, liant dans un seul mouvement l'antiesclavagisme et l'anticolonialisme.

Toutes ces initiatives et publications sont extérieures au contrôle de l'abolitionnisme britannique. De même, la question des libres de couleur est-elle à la fois spécifique et extérieure à la tradition anglaise. En même temps qu'il rétablissait l'esclavage, Napoléon avait rétabli un système ségrégationniste qui refusait la pleine citoyenneté aux libres de couleur. La Restauration a non seulement maintenu ce régime d'apartheid, mais elle l'a encore durci. Pour avoir dénoncé ce régime, un mulâtre de la Martinique, Cyril Bissette, est arrêté avec deux de ses compagnons, Louis Fabien fils et Jean-Baptiste Volny, au début de l'année 1824, et condamné d'abord aux travaux forcés, puis au bannissement, après révision de son procès en 1827 . La cause des hommes de couleur devient celle des libéraux 
et on voit se reconstituer l'alliance contre la ségrégation qui avait joué un rôle considérable pour l'adoption de l'égalité civile pendant la Révolution. L'avocat de gauche, abolitionniste, François Isambert, défend avec succès Bissette et ses compagnons. Une nouvelle génération abolitionniste, la troisième, prend le relais.

La révolution de 1830 et l'arrivée au pouvoir de Louis-Philippe consacrent la victoire de nombre de libéraux abolitionnistes, les plus marquants étant Victor De Broglie, La Fayette, et Guizot. Mais elle inaugure également une période de désillusions et d'impatiences qui va favoriser le passage de relais entre générations. Certes, les lendemains de la révolution libérale voient l'adoption d'une série de mesures qui sont loin d'être négligeables : la traite clandestine, qui n'est plus protégée par le pouvoir, va progressivement disparaître; et les lois de ségrégation sont abandonnées, les libres de couleur pouvant accéder à la pleine citoyenneté (droit de vote, à condition qu'ils en aient les capacités pécuniaires) à partir de l'ordonnance du 24 avril 1833. Mais l'esclavage persiste comme mode de production dominant dans les colonies. L'abolition graduelle généralisée dans les Antilles britanniques à partir de 1833, relance le mouvement en France.

50 Alors que la Société de la morale chrétienne perdure, son comité pour l'abolition de l'esclavage prend son indépendance en 1834, en se transformant en Société française pour l'abolition de l'esclavage, toujours dominée par les libéraux, De Broglie, Tocqueville, Rémusat, Isambert. Indépendance relative toutefois, puisque la tutelle britannique perdure : Zacharie Macaulay et John Scoble, de l'Antislavery Society, sont présents à la fondation. Parmi les membres fondateurs, la continuité l'emporte également : derrière les personnalités dirigeantes, on retrouve Alexandre De Laborde, Hippolyte Carnot, De Gerando, La Rochefoucauld-Liancourt, aux côtés de figures émergentes comme Alphonse De Lamartine, Félix Renouard De Sainte-Croix, ou Destutt De Tracy ${ }^{29}$.

51 Parallèlement, en 1832, Bissette et ses amis fondent à Paris une Société des hommes de couleur, dont l'organe est la Revue des colonies. Avec le guadeloupéen Mondésir Richard et le martiniquais Louis Fabien, Bissette se réclame dans un article de l'abolitionnisme général et graduel; puis, dès 1834, il réclame une abolition immédiate, se démarquant ainsi des positions de la Société pour l'abolition de l'esclavage ${ }^{30}$. Cette dernière a obtenu la nomination d'une commission présidée par son plus illustre représentant, Victor De Broglie, la Commission pour l'examen des questions relatives à l'esclavage et à la condition politique des colonies. Ses travaux durent trois ans (1840-1843). Elle produit un grand nombre d'enquêtes sur la condition des esclaves, et préconise une abolition progressive par rachat (le pécule constitué par les esclaves étant complété par une aide publique) ou une émancipation générale à l'horizon d'une génération (vingt ans), le temps de préparer la substitution de main d'œuvre et l'éducation à la liberté.

\section{Les impasses du gradualisme et la demande d'abolition immédiate. Un exceptionnalisme républicain assumé.}

Les échecs patents de l'abolition graduelle dans les colonies britanniques et les observations enregistrées aux Antilles lors d'une mission d'enquête allaient conduire Victor Schoelcher à rompre avec l'abolitionnisme gradualiste. Il concourt au prix «abbé Grégoire » en 1838 pour un mémoire répondant à la question: Quels seraient les moyens d'extirper le préjugé injuste et barbare des Blancs contre la couleur des Africains et des Sangmêlés? Selon lui, le préjugé de couleur provient de l'esclavage et ne peut être extirpé 
radicalement que par l'abolition immédiate. Le jury estime que l'argumentation ne répond pas à la question posée, mais Schoelcher publie tout de même son mémoire en 1840, puis récidive plus nettement en 1842 avec Des colonies Françaises. Abolition immédiate de l'esclavage. Il y revendique clairement l'héritage révolutionnaire, puis, l'année suivante, l'héritage de la révolution haïtienne dans Colonies étrangères et Haïti ${ }^{31}$.

53 C'est une triple rupture: politique, puisque l'abolitionnisme radical va être une revendication du courant républicain; idéologique, puisque le legs révolutionnaire est accepté en bloc, éloignant et le spectre de la Terreur, et celui de la révolution haïtienne sanglante qui lui sont associés; et géostratégique, puisque l'originalité et l'indépendance de la voie française sont affirmées face aux lenteurs et aux ambiguïtés de la voie britannique.

La presse républicaine s'ouvre aux questions coloniales. C'est particulièrement le cas de La Réforme, journal dirigé par Ledru Rollin, où Schoelcher tient la rubrique coloniale à partir de 1843. Dans la Démocratie pacifique de Victor Considérant, on s'intéresse au système de Jules Lechevalier, qui propose une association des anciens esclaves aux bénéfices de l'entreprise coloniale régénérée (Réorganisation des colonies à esclaves. Émancipation des Noirs combinée avec la libération de la propriété foncière, l'organisation du travail libre et de la colonisation des terres vacantes. Procédé français.). Hippolyte Carnot rédige en 1844 l'article "Esclavage» de L'Encyclopédie nouvelle, où il rappelle la France à son héritage révolutionnaire : «Sa législation, la première en Europe, a prononcé l'abolition de l'esclavage colonial.»

Des campagnes de pétitions sont lancées à travers le pays, recueillant des milliers de signatures, notamment dans les quartiers ouvriers de Paris et dans les bourgs de tradition républicaine. Ainsi, les ouvriers de Paris et de Lyon protestent contre le système de l'esclavage " au nom de la classe ouvrière » et contre ses soutiens qui prétendent que le sort des esclaves est plus enviable que celui des ouvriers européens. La dernière pétition fut rédigée en août 1847, au nom de la Société pour l'abolition de l'esclavage, par Schoelcher : elle réclamait pour la première fois une abolition complète et immédiate. Ainsi, au moment où la ligne des abolitionnistes français républicains s'éloignait de celle des abolitionnistes anglais, elle en adoptait les modes d'action démocratiques. Depuis la fin du XVIII ${ }^{\mathrm{e}}$ siècle, les abolitionniste anglais avaient l'habitude d'appuyer leurs actions sur l'opinion publique en diffusant des brochures, en recueillant des milliers de signatures au bas de leurs textes. Pendant longtemps, le mouvement abolitionniste français avait été élitiste, donnant la priorité à la pression d'un petit noyau de notables influents dans les cercles du pouvoir. Les républicains rompent avec cette orientation élitiste et font de la cause abolitionniste une cause démocratique.

$\mathrm{Au}$ moment où leurs idées s'imposent, les désaccords persistent entre Bissette et Schoelcher, les deux pionniers de l'abolition immédiate ; rivalités personnelles, mais aussi désaccords sur la question religieuse et la question des sang-mêlés. Schoelcher est résolument laïc, voire athée, et reproche à Bissette son spiritualisme, tandis que ce dernier condamne le mépris que Schoelcher témoignerait à l'égard des sang-mêlés, et notamment des femmes. Ce qui ne les empêche nullement de se concurrencer pour la mobilisation du monde protestant, qui manifeste un soutien constant à la cause, ce qui se traduit par des initiatives communes avec le pasteur de Montauban, Guillaume De Felice.

$57 \mathrm{Au}$ cours des dernières années de la monarchie de Juillet, la Société pour l'abolition de l'esclavage sombre dans l'immobilisme. Les propositions de la Commission de Broglie, 
après avoir été présentées devant la Chambre des députés, devaient être reprises par le ministre de la Marine et des colonies, l'amiral Armand De Mackau, dans une série de lois votées en juillet 1845. Les interventions régaliennes de l'État vont prévaloir sur le pouvoir domanial des maitres ${ }^{32}$.

Ces lois Mackau proposaient des aménagements du régime esclavagiste qui n'étaient pas négligeables, et qui auraient pu passer pour «avancés » quelques décennies auparavant. $\mathrm{Au}$ regard de l'évolution des consciences et des revendications, elles paraissent tout simplement dérisoires. Les dispositions les plus marquantes assuraient, sous condition, à l'esclave traduit en justice, la personnalité juridique. Sinon, c'étaient quelques dispositions complémentaires d'un dispositif déjà ancien: les rachats d'esclaves sont facilités, les heures d'instruction religieuse sont obligatoires et étendues, alors que les heures de labeur sont réglementées. Les mariages sont encouragés. Les coups de fouet sont limités à quinze, et les châtiments corporels sont passibles de la justice. Toutefois, aucune réglementation ne peut entrer en vigueur sans l'aval des conseils coloniaux, où les planteurs font la loi. Le gouvernement de Louis-Philippe et de Guizot continue à penser que l'abolition immédiate entraînerait des massacres et la perte des colonies. Le cadre colonial lui-même demeure intact ; au grand dam des abolitionnistes, ces textes n'ouvrent aucunement la voie à une émancipation générale. Seul est prévu un affranchissement collectif et rapide des esclaves du domaine public (les ci-devant « nègres du roi »). Pis, la résistance des colons se crispe et un parti du statu quo esclavagiste fait entendre sa voix jusqu'à l'enceinte de la Chambre des députés et aux bureaux du Ministère.

C'est une nouvelle révolution qui allait imposer la fin de l'esclavage, celle qui mit un terme au régime de la Charte monarchique et qui amena la proclamation de la Seconde République en février 1848.

L'abolition immédiate, dans la tradition de la Grande Révolution, allait s'imposer avec le décret du 27 avril 1848, le retour de la République ayant conduit à la nomination de Schoelcher au poste de sous-secrétaire d'État du ministre de la Marine et des colonies, François Arago, autre abolitionniste radical. Ainsi, l'abolition immédiate, républicaine, pouvait s'imposer dans un contexte autre que celui de la Première République, qui était alors celui d'un état d'exception causé par la guerre. Mais ce ne fut pas sans mal, car il restait à vaincre la résistance des colons, bien organisés. Les révoltes d'esclaves, en mai 1848, avant même la promulgation locale du décret d'émancipation, purent venir à bout de cette résistance.

61 Le 22 mai, une révolte d'esclaves eut lieu au Prêcheur et à Saint-Pierre de la Martinique. Le gouverneur Rostoland proclama le décret le 23 mai. À la Guadeloupe, des rassemblements menaçants d'esclaves se produisirent: le gouverneur Layrle proclama l'abolition le 27 mai. A la Guyane (10 août) et à la Réunion (20 décembre), le délai de deux mois prévu par le décret avant sa promulgation locale fut respecté.

\section{Conclusion}

62 N'est évoqué brièvement dans cette contribution que l'abolitionnisme organisé. Il faudrait encore mentionner un abolitionnisme diffus, partie prenante de la nébuleuse de l'opposition libérale ou bien qui irrigue la conscience publique à travers les productions littéraires et artistiques, comme chez le peintre Géricault, qui revint à plusieurs reprises sur la condition des Noirs, et des esclaves, ou dans la pièce Ourika, de Claire De Duras, la 
fille du député girondin Kersaint, qui date de $1823^{33}$. L'abolitionnisme organisé suffit toutefois à faire mentir le portrait frileux et marginal que l'on propose trop souvent de ce courant d'opinion, artificiellement opposé à l'expression massive et démocratique de l'abolitionnisme britannique.

Les interactions entre les abolitionnismes français et britannique sont à la fois évidentes et contradictoires. La rupture qui s'opère dans les années 1840 entre l'abolitionnisme gradualiste, qui a toujours été la marque des milieux anglo-saxons, et l'abolitionnisme immédiatiste, qui renoue avec le précédent révolutionnaire, est capitale.

L'expérience de la révolution haïtienne est au cœur de ce passé et de cette rupture. Évoquer les origines de Haïti, pour les gradualistes, aurait constitué une gêne, dans la mesure où la conquête de la liberté générale s'est faite dans la conjoncture révolutionnaire. Elle est bien souvent associée à l'évocation de la Terreur ${ }^{34}$.

La relecture de 1793 par les républicains, au cours de ces années 1840, est indissociable de la réévaluation des conditions dans lesquelles Haïti avait acquis son indépendance. La légitimité de cette indépendance n'avait par ailleurs jamais été contestée par les abolitionnistes ${ }^{35}$.

\section{NOTES}

1. La bibliographie de ces mouvements de résistance servile dans le monde atlantique est immense et a déjà été signalée à l'occasion des trois premières rencontres du grand séminaire d'histoire des outremers (Paris, Réunion, Guadeloupe). Nous nous limiterons donc à quelques références :

- Jean fouchard, Les marrons de la liberté, Port-au-Prince, Éditions Henri Deschamps, 1998 (réédition).

- Richard PRICE, Maroons societies. Rebel slave communities in the Americas, Baltimore, John Hopkins University Press, 1979 ( $2^{\mathrm{e}}$ édition).

- Robin BLACKBURN, The overthrow of colonial slavery (1776-1848), Londres, Verso, 1988.

- Prosper ÈVE, Les esclaves de Bourbon, la mer et la montagne, Paris, Karthala, 2003.

2. Ces pics de reprise de la traite après les phases de conflit sont particulièrement nets sur les courbes du rythme de la traite atlantique : p. 27 de Marcel DORIGNY et Bernard GAINOT, Atlas des esclavages, Paris, Autrement, 2013 ( $4^{\mathrm{e}}$ édition).

3. Lawrence JENNINGS, La France et l'abolition de l'esclavage, 1802-1848, Paris, André Versaille éditeur, 2010.

4. Olivier GRENOUILLEAU, La révolution abolitionniste, Paris, Gallimard, 2017, p. 215.

5. Avec une présentation singulièrement réductrice de la réalité ; ainsi, cette autre forme d'évidence contrefactuelle: «Saint-Domingue où les esclaves se sont déjà libérés tous seuls ", Ibid. p. 152.

6. Ibid., p. 215. 
7. Dans cette brève rétrospective du mouvement abolitionniste français, je n'aborderai que superficiellement la place de Haïti, que j'ai plus particulièrement traitée dans un article à paraître de la revue Haitian History Journal/Revue d'histoire haïtienne (Montréal, sous la direction de Carolyn Fick et Franck Voltaire) au printemps 2019, sous le titre: "L'État d'Haïti dans la renaissance du mouvement abolitionniste Français sous la Restauration (1815-1825)».

Voir également sur cette question Yves BÉNOT, «La réception de l'indépendance noire de Haïti en France, de l'abbé De Pradt (1801) à l'abbé Grégoire (1827) » dans Revue de la société haïtienne d'histoire et de géographie, Port-au-Prince, $n^{\circ}$ 217/2004. Du même auteur, « Haïti et la revue encyclopédique" dans Les Lumières, l'esclavage, la colonisation, Paris, La Découverte, 2005, p. 273-283.

8. Ce texte vient d'être traduit en français, pour la première fois, sous la direction de Marie-Jeanne RosSIGNOL et Bertrand VAN RUYMBECKE, Une histoire de la Guinée, Publications de la Société française d'histoire du XviII ${ }^{\mathrm{e}}$ siècle, 2017.

9. Pour illustrer ce courant anti-esclavagiste chrétien précoce, qui se nourrit du monogénisme de l'école de Salamanque, on peut renvoyer à l'ouvrage du Père jésuite CLAVER, Naturaleza policia sagrada y profana, costumbres y ritos, disciplina y catecismo evangelico de todos los Etiopes, Séville, 1647. Pour replacer la question dans son contexte, je conseille l'essai d'Hélène VIGNAUX, L'Église et les Noirs dans l'audience du Nouveau Royaume de Grenade, Montpellier, Presses universitaires de la Méditerranée, 2009.

10. Jean EHRARD, Lumières et esclavage. L'esclavage colonial et la formation de l'opinion publique en France au XVIII ${ }^{\mathrm{e}}$ siècle, Bruxelles, André Versaille, 2008.

11. Pernille ROGE, « 'La clef de commerce'-The changing role of Africa in France's Atlantic empire ca. 1760-1797 » dans History of European ideas, 34/2008, p. 431-443.

Marcel DoRIGNY ; « La Société des Amis des Noirs et les projets de colonisation en Afrique » dans Annales historiques de la Révolution française, numéro spécial "Révolutions aux colonies ", nº 293-294/1993, p. 421-429.

12. Voir la publication récente de la journée d'étude organisée par l'Association pour l'étude de la colonisation européenne (1750-1850) - Apece : Guillaume Thomas Raynal: les colonies, la Révolution française et l'esclavage, Société française d'histoire de l'outre-mer, 2015.

13. Marcel DORIGNY et Bernard GAINOT, La Société des Amis des Noirs, 1788-1799: Contribution à l'histoire de l'abolition de l'esclavage, Paris, Publications de l'Unesco, 1997.

14. La plus marquante était Helen-Maria Williams; voir Marcel DoRIGNY et Bernard GAINOT , La Société des Amis des Noirs..., op.cit. p. 299-396.

15. Sur cet aspect, on se reportera aux actes du colloque Couleur, esclavages, libérations nationales (1804-1860), sous la direction de Claire Bourhis-Mariotti, Bernard Gainot et Clément Thibaud, Paris, les Perséides, 2013; notamment les contributions de Bernard GAINOT (p. 105-120), de Suzanne schWARz (p. 177-200), de Claire BOURHIS-MARIOTTI (p. 221-242), de Joseph yanielli (p. 243-262.) Sur cet ensemble de projets qui émaillent cette période de transition, regroupés sous le nom de "colonisation nouvelle ", voir la synthèse récente La colonisation nouvelle, Marcel DORIGNY et Bernard GAINOT dir., Paris, SPM, 2018. Elle reprend une journée d'études organisée par l'APECE sur ce thème en juin 2016. 
16. Lionel TRANI, La Martinique napoléonienne, 1802-1809. Entre ségrégation, esclavage et intégration, Paris, Éditions SPM, 2014.

Bernard GAINOT, "The Empire overseas: the illusion of Restoration " dans Napoleon's Empire, European politics in global perspective, sous la direction de Ute Planert, Palgrave Macmillan, 2015 .

Jean-François NIORT (dir.), Du Code Noir au Code civil, Jalons pour l'histoire du droit en Guadeloupe. Paris, L'Harmattan, 2007.

17. Yves BÉNOT, La démence coloniale sous Napoléon, Paris, La découverte, 1991. Voir également, de Lawrence JENNINGS, Abolitionnisme, jeu politique et réforme: France, 1814-1848, in Olivier Pétré-Grenouilleau (dir.), Abolir l'esclavage: un réformisme à l'épreuve : France, Portugal, Suisse, XVIII ${ }^{\mathrm{e}}$-XIX ${ }^{\mathrm{e}}$ siècles, Rennes, PUR, 2008, p. 169-184.

Du même auteur, La lente renaissance du mouvement abolitionniste en France, in Yves Bénot et Marcel Dorigny (dir.), Rétablissement de l'esclavage dans les colonies françaises, 1802: Rupture et continuité de la politique coloniale française (1800-1830), Paris, Maisonneuve et Larose, 2003, p. 365-374.

18. Numéro spécial des Cahiers staëliens, $\mathrm{n}^{\circ} 64 / 2014$, Le groupe de Coppet face à l'esclavage, notamment Marcel DORIGNY, "Sismondi, de la lutte contre la traite à la colonisation nouvelle : les combats d'un intellectuel cosmopolite au début du XIX ${ }^{\mathrm{e}}$ siècle », p. 77-92.

19. Le Congrès de Vienne et les traités de 1815, Paris, Amyot, Éditions des archives diplomatiques ; cité par Nelly SCHMIDT, L'abolition de l'esclavage. Cinq siècles de combats XVI ${ }^{e}$ $X^{e}$ siècles, Paris, Fayard, 2005, p. 125. Voir encore la thèse récente de Michel ERPELDING, sous la direction de Emmanuelle Tourme-Jouannet, université Paris 1 PanthéonSorbonne, Le droit international antiesclavagiste des nations «civilisées » (1815-1945) (soutenue en 2017).

20. Emblématique de ce courant est la publication du texte du colon Antoine Dalmas, émigré aux États-Unis en 1793, Histoire de la révolution de Saint-Domingue : depuis le commencement des troubles... suivie d'un mémoire sur le rétablissement de cette colonie, paru chez Mame, à Paris, en 1814. Sur l'importance de ce "moment 1814 ", voir Yves BÉNOT, La démence coloniale sous Napoléon, op.cit., p. 205-208.

21. Serge DAGET, La traite des Noirs, Rennes, Éditions Ouest-France, 1990, et, du même auteur, La répression de la traite des Noirs au XIX ${ }^{\mathrm{e}}$ siècle. L'action des croisières françaises sur les côtes occidentales de l'Afrique, Paris, Karthala, 1997.

22. Lawrence C. JENNINGS, La France et l'abolition de l'esclavage 1802-1848, André Versaille éditeur, 2010 (traduction française de French anti-slavery. The movement for the abolition of slavery in France, 1802-1848, Cambridge University press, 2000).

23. Catherine DUPRAT, Usages et pratiques de la philanthropie. Pauvreté, action sociale et lien social, à Paris, au cours du premier XIxe siècle, Paris, Comité d'histoire de la Sécurité sociale, 1996, tome 1, Protestants et libéraux. La Société de la morale chrétienne.

24. Joseph-Elzéar MORENAS, Pétition contre la traite des Noirs qui se fait au Sénégal, présentée à la Chambre des députés le 14 juin 1820, Paris, Corréard, 1820 ; et, du même, Seconde pétition contre la traite des Noirs présentée à la Chambre des députés le 21 mars 1821, toujours chez Corréard, 1821.

25. Général Pamphile DE LACROIX, La Révolution de Haïti, Paris, Karthala, 1995 (réédition de l'ouvrage de 1819, Mémoires pour servir à l'histoire de la révolution de Saint-Domingue). 
26. Antoine MÉTRAL, Histoire de l'expédition des Français à Saint-Domingue sous le Consulat de Napoléon Bonaparte (1802-1803), Paris, Karthala, 1985 (réédition de l'ouvrage original de 1825).

27. Colonel malenfant, Des colonies, et particulièrement de celle de Saint-Domingue, Paris, août 1814.

28. CIVIQUE DE GASTINE, Histoire de la république d'Haïti ou Saint-Domingue: l'esclavage et les colons, Paris, Plancher, 1819. On remarque que l'ouvrage sort des mêmes presses que ceux de Morénas, l'imprimerie de Mme Jeune-homme Crémière, indice d'un courant radical, extérieur à la Société de la morale chrétienne.

29. Patricia MOTYLEWSKI, La Société française pour l'abolition de l'esclavage (1834-1850), Paris, L'harmattan, 1998.

Philippe VIGIER, «La recomposition du mouvement abolitionniste français sous la Monarchie de Juillet » in Marcel Dorigny (dir.), Les abolitions de l'esclavage de Sonthonax à Schoelcher, 1793, 1794, 1848, Presses universitaires de Vincennes/Éditions Unesco, 1995, p. 285-291.

30. Nelly SCHMIDT, L'abolition de l'esclavage..., op.cit. p. 207.

31. Anne GIROLLET, Victor Schoelcher, abolitionniste et républicain. Approche juridique et politique de l'œuvre d'un fondateur de la République, Paris Karthala, 2000.

Nelly SCHMIDT, Victor Schoelcher, Paris, Fayard, 1994 (réédition 1999).

Seymour DRESCHER, "British way, French way: opinion building and revolution in the second French slave emancipation ", in S. Drescher, From slavery to freedom: comparative studies in the rise and fall of Atlantic slavery, Hampshire, Macmillan press, 1999, p. 158-195.

32. Caroline OUDIN-BASTIDE, Maîtres accusés, esclaves accusateurs. Les procès Gosset et Vivié, Marinique, 1848, Presses universitaires de Rouen et du Havre, 2015.

33. Claire DE DURAS, Ourika, Paris, Imprimerie Royale, 1823. Parme les très nombreuses rééditions récentes, j'ai choisi celle de Folio/Gallimard, 2010.

34. Olivier GRENOUILLEAU, La révolution abolitionniste, p. 215: «l'abolitionnisme fut en France rapidement associé par ses opposants à l'idée de Terreur et à la perte de SaintDomingue attribuée aux propos des abolitionnistes. D'où un divorce entre abolitionnisme et idée nationale, mais aussi les réticences des milieux abolitionnistes ultérieurs vis-à-vis de l'action populaire. » On relève d'autres formes d'évidence contrefactuelle, ainsi p. 152 : "Saint-Domingue où les esclaves se sont déjà libérés tous seuls ». Le propos est toujours de relativiser l'exceptionnalité de la parenthèse républicaine 1794-1799.

35. «L'État d'Haïti dans la renaissance du mouvement abolitionniste Français sous la Restauration (1815-1825) », Haitian History Journal, op.cit.

\section{RÉSUMÉS}

Le mouvement pour l'abolition de l'esclavage se développe principalement, mais pas exclusivement, au sein des opinions publiques des deux principales puissances coloniales 
concernées, l'Empire britannique et l'Empire français. Mais c'est surtout l'abolitionnisme anglosaxon qui impose sa marque et son agenda, une abolition très graduelle, qui cherche prioritairement une interdiction de la traite négrière par tous les pays occidentaux concernés. Les aléas du processus révolutionnaire, tout particulièrement à Saint-Domingue, et la guerre entre la France et l'Angleterre, vont toutefois bouleverser le programme. Une autre voie apparait, l'abolition immédiate, directement portée par les populations de couleur.

Après la phase du rétablissement de l'esclavage par le régime napoléonien, c'est le gradualisme qui revient à l'ordre du jour. Mais les lenteurs et les déconvenues du processus (interdiction de la traite en 1807, abolition graduelle à partir de 1833) dans les colonies britanniques, ainsi que l'évolution de l'attitude envers l'héritage de la Révolution Française, conduisent à un retour en force de l'immédiatisme. À partir de 1843 en France, c'est la revendication immédiate qui s'impose, couplée à des campagnes massives de pétitions dans le monde ouvrier, de sensibilité républicaine.

The development of the abolition movement occurred mostly, but not exclusively, in the public opinions of the two main colonial powers, the British and French Empires. But it was the British abolitionism that would prevail and impose its agenda, one promoting a very gradual abolition, essentially by having all involved western nations prohibiting the African slave trade. However, the vagaries of the revolutionary process, particularly in Saint-Domingue, and the war between France and England would disturb this programme. Another way would emerge, immediate abolition, supported directly by the coloured populations.

After the Napoleonian regime restored slavery, gradualism became again the path to follow. But the process' slowness and disappointing results (prohibition of the slave trade in 1807, partial abolition beginning in 1833) in the British colonies, as well as the evolving attitude towards the French Revolution's heritage, led to a strong come-back of "immediatism". After 1843, in France, the demand for an immediate reform became dominant, coupled with massive petition campaigns across the working class, of Republican leaning.

\section{INDEX}

Mots-clés : abolition graduelle, abolition immédiate, révolutions coloniales, traite négrière, libres de couleur, réformisme ministériel

Keywords : gradual abolition, immediate abolition, colonial revolutions, African slave trade, free people of colour, ministerial legalism

\section{AUTEUR}

\section{BERNARD GAINOT}

IHMC-IHRF

université Paris 1 Panthéon-Sorbonne 